\title{
Endoscopic Solution to Rhinogenic Contact Headaches
}

\author{
Hasan Abdul Cader Segana, ${ }^{1}$ Reghunandanan Nair, ${ }^{1}$ Fahim Ahmed Shah ${ }^{1}$
}

\section{Introduction}

\section{ABSTRACT}

Headache is a common complaint that brings patients to multidisciplinary clinics. It is utmost important to have meticulous clinical diagnosis of patients with rhinogenic and non sinusogenic headaches. The diagnosis has become easier with the advent of modern endoscopy and endoscopic sinus surgical techniques. This study aims to investigate the role of some anatomical nasal abnormalities in rhinogenic contact headache and to evaluate response to endoscopic surgery.

\section{Materials and Methods}

A prospective study was conducted at a secondary level regional referral Hospital in the Sultanate of Oman. Patients with longlasting, frequent, severe headaches not amenable to medical treatment, above 20 years of age were taken into consideration. Routine nasal endoscopy, Computerized tomography scan of the paranasal sinuses, Nasal decongestion and various surgical techniques to correct the anatomical abnormalities were included in our study and results were correlated statistically.

$\underline{\text { Result }}$

There was a male predominance in our study with duration of headache ranging from 2 weeks to 5 years. There was a preponderance of headache in frontal region in our study group. Diagnostic nasal endoscopy and CT scan of PNS revealed Deviated nasal septum / septal spur, concha bullosa, Haller cell, pneumatised uncinate process and agar nasi cells. The overall success rate of the surgery in relieving headaches, measured by the MIDAS-VAS score, was approximately $75 \%$. The nonparametric Wilcoxon signed rank test, Chi square and paired T tests shows that the following study has rejected the null hypothesis as statistically significant where the P value $<0.05$.

\section{Discussion}

Researchers have examined the contact points as a source of rhinogenic / contact headache. Intranasal mucosal contact released substance P, causing pain and headache, Substance P has a potent vasodilator effect. Vasodilatation and perivascular inflammation are the final common pathways in pain. Surgical treatment for contact point-induced headaches has had good success.

\section{Conclusion}

The etiology of rhinogenic headache is multifactorial. Complete history taking, scrupulous preoperative evaluations, multidisciplinary consultations, Initial medical controls, long observation, and diligent postoperative follow-ups are mandatory for not only accurate diagnosis but also for promising surgical outcomes of non-sinusitis related rhinogenic headache. Our experience reveals that patients with rhinogenic contact headaches can benefit significantly from meticulous endoscopic decompression

Kevwords:

Headache; Facial Pain; Forehead; Endoscopy; Nasal Mucosa; Contact Points; Surgical Procedures, Operative

$\mathrm{H}$ eadache is a common complaint flocking patients to General Physicians, Neurologists, Ophthalmologists and otolaryngologists in date to day clinical practice. It's therefore mandatory to have high proficiency and meticulous care in evaluating

\section{1 - Sur Hospital, Sur, Oman}

\section{Corresponding author:}

Hasan Abdul Cader Segana

email: dr_shac@yahoo.com headaches of both rhinologic and non sinusogenic origin. Rhinogenic or sinusogenic headache is a controversial topic to be discussed, but this is gaining increasing attention following advent of endoscopic solution during last two decades. This study aimed to investigate the role of some anatomical nasal abnormalities in rhinogenic contact headache and to evaluate response to endoscopic surgery. It also defines rhinogenic headache, and reviews the literature supporting and opposing the existence of rhinogenic headaches.

The concept of rhinogenic headache secondary to 
mucosal contact points was first described by J.O. Roe in $1888 .{ }^{1}$ In the 1920 's, Sluder theorized that headaches could occur from the sinuses in the absence of inflammation or infection by the creation of a vaccum in a sinus cavity. McAuliffe et. al. performed a frequentlysited study in 1943 regarding the origin of headache and facial pain. Both the International Headache Society (IHS) and the American Academy of OtolaryngologyHead and Neck Surgery (AAO-HNS) have described conditions that can cause headaches of rhinogenic origin. $^{2}$

Rhinogenic headache is secondary to mucosal contact points in the nasal/sinus cavities in the absence of inflammatory sino-nasal, purulent discharge, sino-nasal polyps, sino-nasal masses or hyperplastic mucosa. It has multiple synonyms used frequently in the literature which include rhinopathic headache, sinogenic headache, middle turbinate headache, nasal spur headache, four finger headache, sinus headache, contact point headache, and Sluder headache.

Stammberger and Wolfe described a potential mechanism for nasal mucosal contact point headaches in 1988. According to their theory mechanical contact between two mucosal surfaces in the nose or sinuses creates a sensory stimulus which is known as axonal reflex. Sensory nerve endings primarily from V1 and $\mathrm{V} 2$ extend into the nasal mucosa and nearly to the cell surface. The axonal reflex results in the release of substance $\mathrm{P}$, a vasoactive neuropeptide found in unmyelinated $C$ fibers. ${ }^{3}$ Substance $P$ causes vasodilation, plasma extravasation, histamine release, and other inflammatory events. These vascular phenomenon may be responsible for migraine-like headache symptoms. Since V1 innervates the dura, this mechanism may also involve the above discussed referred pain from V2/V3 as well.

\section{Materials and Methods}

This was a prospective study which was conducted at ENT department in Secondary level regional referral Ministry Hospital in Sultanate of Oman between January 2014 and January 2015.We reviewed those who were accessible for 6 months to 1 year follow up in treatment. 20 such patients were enrolled in our prospective study.

\section{$\underline{\text { Inclusion criteria }}$}

Patients who were above 20 years of age and had long-lasting, frequent, severe headaches without other identifiable sino-nasal disease or other known obvious cause and not relieved by any analgesic treatment.

\section{Exclusion criteria}

Any patient with sinonasal tumour, patients with other obvious causes of headache like migraine, cluster headache, headache caused by ophthalmological causes, patients who refused surgery or follow up care, pregnant ladies and patients with history of previous sinonasal surgeries were excluded from this study.

Preoperatively, all patients were assessed by a questionnaire on the detailed history of their headache including onset, time of day, duration, frequency, nature, side of the head and location, existence of an aura, aggravating and relieving factors, and accompanying nasal symptoms, they were evaluated using a MIDAS [Migraine Disability Assessment Questionnaire Score] Headache score questionnaire based on the activities of daily life (Fig. 1).

All the patients under this study underwent a preliminary diagnostic nasal endoscopy and a CT PNS evaluation to identify nasal mucosal contact points and to rule out other nasal pathologies (Table I). Most of them underwent a nasal decongestion test. Using this test, topical anaesthetic was applied to a mucosal contact point while a patient was actively experiencing headache. A positive test occurred when application of the anaesthetic relieved the headache. Each patient was also evaluated by a multi-disciplinary team consisting of a neurologist, ophthalmologist, orthopaedic specialist, and internist to rule out other possible causes of headaches. The patients were managed initially by medical therapy without success.

Surgically corrected contact points in these case series included deviated nasal septum in contact with 
lateral nasal wall, deviated nasal septum in contact with middle turbinate, deviated septum in contact with inferior turbinate, Pneumatized Middle turbinate or concha bullosa, pneumatised Haller cells, Onodi cells, pneumatised uncinate process and any other visualized mucosal contact point.

\section{Surgical Technique}

After obtaining written informed consent, surgery was performed under general anaesthesia. At the end of the surgical procedure, the nasal cavity was packed with (polyvinyl alcohol formaldehyde foam) Merocel ${ }^{\circledR}$ and gauze which were removed on first postoperative day. Antibiotics and analgesics were administered for 7 days following surgery.

The surgery was done meticulously preserving most of the functional mucosa and it was a limited endoscopic sinus surgery based on location of the anatomical variation, contact points, as identified by nasal endoscope and CT PNS. In patients with nasal septum deviation or septal spur, a septoplasty or spurrectomy was performed initially. The concha bullosa in the diseased side was approached by way of lateral lamellectomy. For Haller cell, the inferior, medial, and lateral walls were carefully removed to fully open the cavity of the cell, with preservation of the superior wall to prevent injury to the orbital cavity. For turbinate hypertrophy turbinoplasty or conservative partial turbinectomy was done on patients with severe hypertrophy of the inferior turbinate.

Postoperative follow-up evaluations were scheduled at 2 weeks, 1, 2, 3, and 6 months. Postsurgical wound care included regular debridement- extirpation of any granulation tissue, post synechiae release and medical therapy The evaluations included an endoscopic examination of the nasal cavity and VAS-MIDAS score grading of headache intensity (Fig. 1). For further analysis patients were asked to grade the pain intensity on visual analog scales, with 0 indicating no pain at all and 10 indicating worst possible pain. The VAS scores of the four groups based on the MIDAS score severity of headache (Table II) were compared using a nonparametric Wilcoxon signed rank test (Table III). The SPSS 16.0 software (SPSS Inc., Chicago, IL) was used to conduct the statistical tests. $p$ values $<0.05$ (2-sided) were deemed to indicate statistical significance.The Ethical research committee of the hospital approved this study protocol .

\section{Results}

Out of the 20 patients accepted for study 12 [60\%] were Female and $8[40 \%]$ were Male patients. The duration of headache was ranging from 2 weeks to 5 years. The mean headache duration was 6.20 hour per headache, ranging from 60 seconds to 24 hour, and the frequency of headaches ranged from one episode per month to every day. The headache occurred in the frontal area in 8 patients, in glabellar region in 5 patients, periorbital in 2 cases, one each in vertex, parietal, temporal, forehead and malar regions. (Table I)

Diagnostic nasal endoscopy and CT scan of PNS revealed deviated nasal septum / septal spur in 8 patients, concha bullosa in 5 cases, Haller cell in 2 patients, pneumatised uncinate process and agar nasi cells in 2 cases and Onodi cell in a patient. Some patients had combination of the above. Timing of headache was another factor - 11 patients had an early morning headache and 9 of them had an evening bout of headache.10 patients had stabbing quality and 4 patients attributed as squeezing and rest 6 patients had a dull vague nature of ache.

The overall success rate of the surgery in relieving headaches, measured by the MIDAS-VAS score, was approximately $75 \%$. Almost complete relief from headaches was achieved in 15 of 20 patients $(75 \%)$, and 3 patients $(15 \%)$ reported a decrease in headache intensity after surgery. In contrast, 2 patients reported that headache intensity was unchanged after surgery $(5 \%)$,or either worsened or remained the same $(5 \%)$. (Table II) The non-parametric Wilcoxon signed rank test, Chi square and paired $\mathrm{T}$ tests shows that the following study has rejected the null hypothesis as statistically significant where the $\mathrm{P}$ value $<0$ 0.05.(Table III)

\section{Discussion}

Many researchers and studies have examined the contact 


\section{midas Questionnaire}

INSTRUCTIONS: Please answer the following questions about ALL. your headaches you have had over the last 3 months. Write your answer in the box next to each question. Write zero if you did not do the activity in the last 3 months.

1 On how many days in the last 3 months did you miss work or school because of your headaches? $\square$ days

2 How many days in the last 3 months was your productivity at work or school reduced by half or more because of your headaches? (Do not include days you counted in question 1 where you missed work or school)

3 On how many days in the last 3 months did you not do household work because of your headaches?

4 How many days in the last 3 months was your productivity in household work reduced by half or more because of your headaches? (Do not include days you counted in question 3 where you did not do household work)

5 On how many days in the last 3 months did you miss family, social or leisure activities because of your headaches?

A On how many days in the last 3 months did you have a headache? (If a headache lasted more than 1 day, count each day)

B On a scale of $0-10$, on average how painful were these headaches? (Where $0=$ no pain at all, and $10=$ pain as bad as it can be)

Onnovative Medical Research 1997

Once you have filled in the questionnaire, add up the total number of days from questions 1-5 (ignore A and B).

\begin{tabular}{|c|c|c|}
\hline Grade & Definition & Score \\
\hline I & Minimal or infrequent disability & $0-5$ \\
\hline II & Mild or infrequent disability & $6-10$ \\
\hline III & Moderate disability & $11-20$ \\
\hline IV & Severe disability & $21+$ \\
\hline
\end{tabular}

Fig.1. Migraine Disability Assessment Questionnaire

points as a source of rhinogenic / contact headache. Stammberger and Wolff suggested that intranasal mucosal contact released substance $\mathrm{P}$, causing pain and headache. Substance P, which is associated with the inflammatory process, has a potent vasodilator effect.
Vasodilatation and perivascular inflammation are the final common pathways in pain. Surgical treatment for contact point-induced headaches has had good success. Clerico et al and Tosun et al has reported an $79 \%$ and $90 \%$ success rate respectively following surgery to 
Table I : Contact areas within nasal cavity that is responsible for headache as confirmed during diagnostic nasal endoscopy by the patch and probe test and CT Scan PNS findings

\begin{tabular}{|c|c|c|c|c|c|c|c|c|c|}
\hline$\stackrel{\ominus}{Z_{i}}$ & 过 & $\frac{x}{\infty}$ & 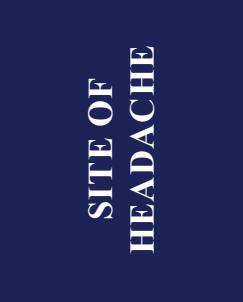 & 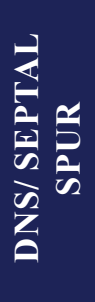 & 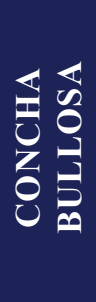 & 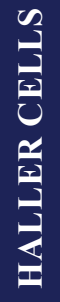 & 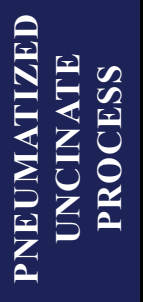 & 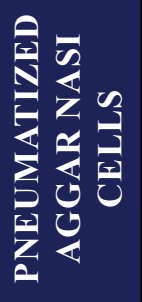 & $\frac{0}{3}$ \\
\hline 1 & 23 & $\mathrm{~F}$ & FRONTAL & 1 & & & & & \\
\hline 2 & 35 & $\mathrm{~F}$ & FRONTAL & 1 & & & & & \\
\hline 3 & 40 & $\mathrm{~F}$ & FRONTAL & & & & 1 & & \\
\hline 4 & 32 & $\mathrm{M}$ & GLABELLAR & & & & & 1 & \\
\hline 5 & 26 & $\mathrm{~F}$ & FOREHEAD & 1 & & & & & \\
\hline 6 & 22 & M & TEMPORAL & 1 & & & & & \\
\hline 7 & 36 & $\mathrm{M}$ & MALAR & & 1 & & & & \\
\hline 8 & 32 & $\mathrm{M}$ & FRONTAL & & & & & 1 & \\
\hline 9 & 30 & $\mathrm{~F}$ & FRONTAL & & & & 1 & & \\
\hline 10 & 27 & M & PERIORBITAL & & 1 & & & & \\
\hline 11 & 22 & $\mathrm{~F}$ & GLABELLAR & 1 & & & & & \\
\hline 12 & 24 & $\mathrm{~F}$ & GLABELLAR & 1 & & & & & \\
\hline 13 & 27 & $\mathrm{M}$ & FRONTAL & & 1 & & & & \\
\hline 14 & 31 & $\mathrm{M}$ & PERIORBITAL & & & 1 & & & \\
\hline 15 & 38 & $\mathrm{M}$ & GLABELLAR & & 1 & & & & \\
\hline 16 & 36 & $\mathrm{M}$ & FRONTAL & 1 & & & & & \\
\hline 17 & 27 & $\mathrm{~F}$ & FRONTAL & & 1 & & & & \\
\hline 18 & 21 & $\mathrm{M}$ & VERTEX & & & & & & 1 \\
\hline 19 & 29 & $\mathrm{M}$ & PARIETAL & 1 & & & & & \\
\hline 20 & 34 & $\mathrm{M}$ & GLABELLAR & & & 1 & & & \\
\hline
\end{tabular}

correct contact point induced headaches. The following table represents an overview of different authors in successfully relieving the contact headaches including one of our own study as well. Table IV depicts an overview of different authors in successfully relieving the contact headache with different surgical techniques they used.

Mohebbi et al reported $83 \%$ success rate following surgery to correct contact point-induced headaches. ${ }^{8}$ Stammberger and Wolf suggested that hypoxia 
Table II : Preoperative and Postoperative VAS-MIDAS Questionnaire

\begin{tabular}{|c|c|c|}
\hline MIDAS SCORE SEVERITY OF HEADACHE & $\begin{array}{c}\text { PREOPERATIVE } \\
\text { PAIN SCORE }\end{array}$ & $\begin{array}{c}\text { POSTOPERATIVE } \\
\text { PAIN SCORE }\end{array}$ \\
\hline 0-5(Minimal or infrequent disability- Grade I) & 2 & 15 \\
\hline 6-10(Mild or infrequent disability- Grade II) & 4 & 3 \\
\hline 11-20(Moderate disability- Grade III) & 12 & 1 \\
\hline$>21$ (Severe Disability- Grade IV) & 2 & 1 \\
\hline
\end{tabular}

Table III : Statistical Analyses

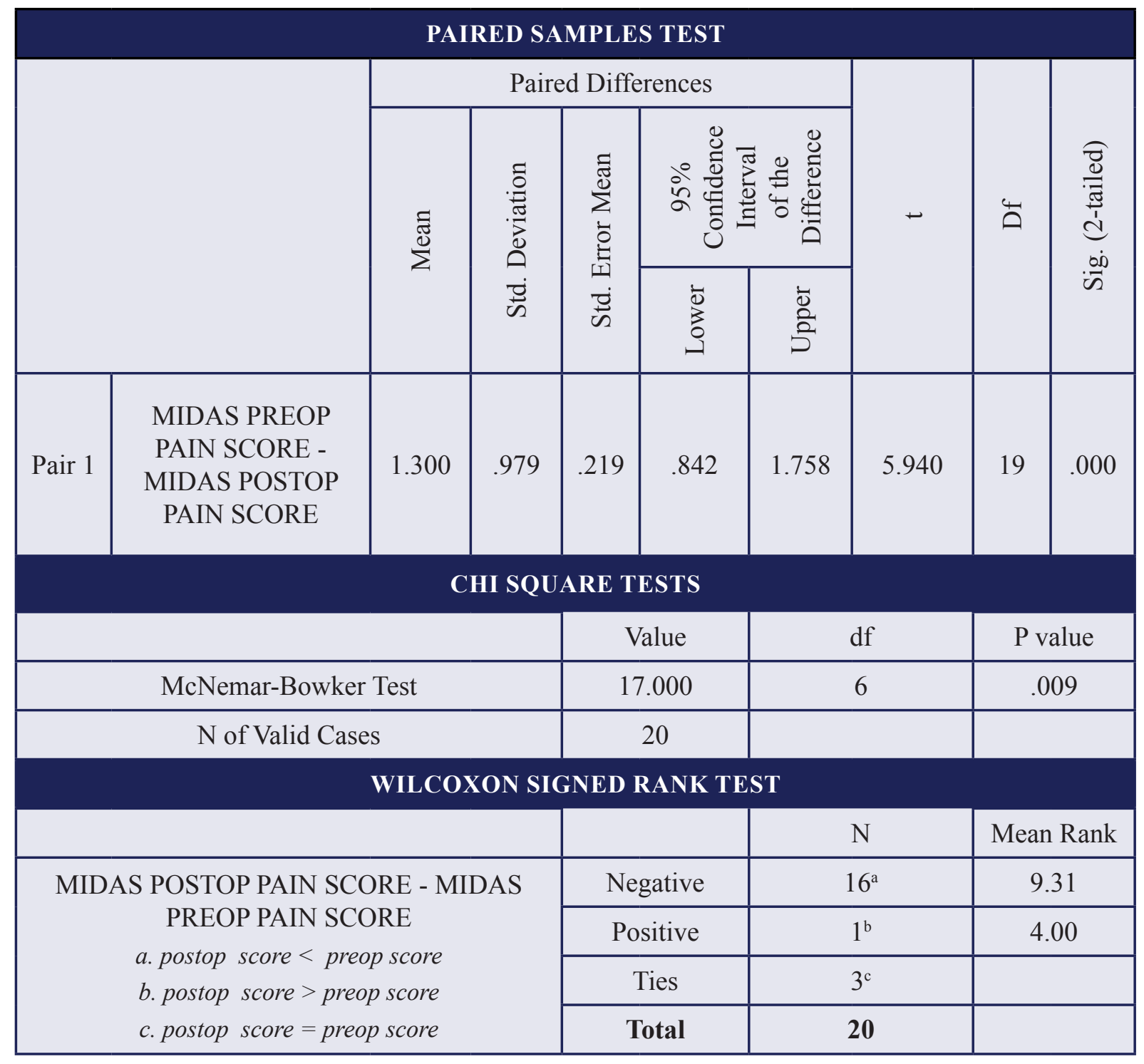


Table IV : An overview of different studies reporting successful treatment of contact headache

\begin{tabular}{|c|c|c|c|c|}
\hline 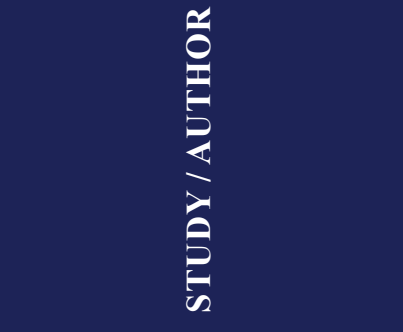 & $\frac{\mathscr{D}}{\underset{E}{Z}}$ & $\sum_{\infty}^{\infty}$ & 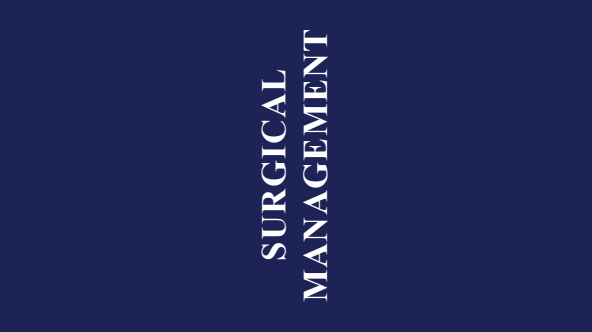 & 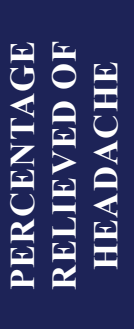 \\
\hline Schonsted-Madsen et al ${ }^{4}$ & 157 & Headache & $\begin{array}{l}\text { septoplastic surgery, reconstruction } \\
\text { of the nasal pyramids, or submucosal } \\
\text { conchotomy }\end{array}$ & $60 \%$ \\
\hline Low and Willatt et al ${ }^{5}$ & 116 & Headache & $\begin{array}{c}\text { submucous resection for a deviated } \\
\text { nasal septum }\end{array}$ & $63 \%$ \\
\hline Parsons and Batra et al ${ }^{6}$ & 34 & Headache & FESS & $91 \%$ \\
\hline Ramadan et al $^{7}$ & 23 & Headache & FESS & $60 \%$ \\
\hline Our study & 20 & Headache & $\begin{array}{l}\text { ESS-Septoplasty/spurrectomy,lateral } \\
\text { lamellectomy }\end{array}$ & $75 \%$ \\
\hline
\end{tabular}

secondary to pressure differentials within the sinuses was a possible mechanism for the release of substance $\mathrm{P}$.

\section{Conclusion}

The etiology of rhinogenic headache is multifactorial. ${ }^{9}$ In addition to the often-seen anatomical abnormalities, other variations have also been identified as sources of referred pain, for example, paradoxical middle turbinates or overexpanded agger nasi cells. Complete history taking, scrupulous preoperative evaluations, multidisciplinary consultations, initial medical controls, long observation, and diligent postoperative follow-ups are mandatory for not only accurate diagnosis but also for promising surgical outcomes of non sinusitis related rhinogenous headache.

Few prospective studies have investigated the improvement of headaches after nasal or sinus surgery. Mariotti and Setliff evaluated patient history and computed tomography (CT) scans in a prospective study designed to predict the outcome of headaches after surgery. ${ }^{10}$ Our experience reveals that patients with rhinogenic contact headaches can benefit significantly from meticulous endoscopic decompression.

\section{References}

1. Roe JO. The frequent dependence of persistent and so-called congestive headaches upon abnormal conditions of the nasal 
passages. Med Record 1888;34:200-4

2. Cady RK, Dodick DW, Levine HL, Schreiber CP, Eross EJ, Setzen M, et al. Sinus headache: A neurology, otolaryngology, allergy, and primary care consensus on diagnosis and treatment. Mayo Clin Proc. 2005; 80:908-16

3. Lundblad L, Lundberg JM, Brodin E, et al. Origin and distribution of capsaicin-sensitive substance P-immunoreactive nerves in the nasal mucosa. ActaOtolaryngol. 1983;96:485-93

4. Schonsted-Madsen U, Stoksted P, Christensen PH, KochHenriksen N. Chronic headache related to nasal obstruction. J Laryngol Otol. 1986; 100:165-70

5. Low WK, Willatt DJ. Headaches associated with nasal obstruction due to deviated nasal septum. Headache 1995;35:404-6
6. Parsons DS, Batra PS. Functional endoscopic sinus surgical outcomes for contact point headaches. Laryngoscope 1998; 108:696-702

7. Ramadan HH. Nonsurgical versus endoscopic sinonasal surgery for rhinogenic headache. Am J Rhinol. 1999;13:455-7

8. Mohebbi A, Memari F, Mohebbi S. Endonasal endoscopic management of contact point headache and diagnostic criteria. Headache 2010; 50:242-8

9. Acquardro MA. Treatment of chronic paranasal sinus pain with minimal sinus disease. Ann Otol Rhinol Laryngol. 1996;105:607-61

10. Mariotti LJ, Setliff RC, Ghaderi M, Voth S. Patient history and CT findings in predicting surgical outcomes for patients with rhinogenic headache. Ear Nose Throat J 2009;88:926-9 